\title{
Detection of hepatitis B pre-core mutant by allele specific polymerase chain reaction
}

\author{
E S-F Lo, Y-M D Lo, C H Tse, K A Fleming
}

\begin{abstract}
Aim: Development of a specific polymerase chain reaction (PCR) assay for detection of the pre-core, stop codon, mutant of hepatitis B virus (HBV).
\end{abstract}

Methods: PCR primers, specific at the 3 '-end for nucleotide 1896 of either the pre-core, stop codon, mutant or wild type HBV, were synthesised using published sequence data. Positive control templates for both types of virus were synthesised by the PCR, incorporating sequences specific for each virus type at the appropriate position. These templates were used to optimise the specificity of the procedure. Formalin fixed, paraffin wax embedded human tissue from acute or fulminant HBV hepatitis from Hong Kong or Oxford was then investigated for presence of mutant or wild type virus. The HBV DNA was amplified from this tissue using a two step procedure, with an initial amplification phase followed by a second diagnostic phase on optimally diluted target DNA. Results: Specific detection of mutant or wild type HBV was achieved. An important factor in determining specificity was the temperature of annealing, $70^{\circ} \mathrm{C}$ proving to be highly specific. To overcome the inherent variation of target copy number in clinical samples and to provide an intrinsic positive control, it was important to generate and standardise the amount of target HBV used for the specific PCR. Two cases of fulminant hepatitis and four cases of acute hepatitis from Hong Kong, and one case of fulminant hepatitis from Oxford, contained only wild type HBV, with no evidence of a mutant virus.

Conclusion: This method can be applied to FFPE tissues. It is rapid, non-radioactive, and specific for the stop codon mutation at nucleotide 1896 of HBV. Preliminary investigation of a small number of cases of fulminant hepatitis from Oxford and Hong Kong showed only wild type virus. The result differs from results published from Japan and Israel.

Over the past few years, development of molecular biological techniques, including the polymerase chain reaction (PCR), ${ }^{1}$ has resulted in the detection of pre-core mutant forms of hepatitis B virus (HBV). ${ }^{23}$ The most common pre-core mutation is a G-A substitution found at nucleotide 1896 (nomenclature according to Galibert et $a l^{4}$ in the pre-core open reading frame (ORF) which results in the generation of a stop codon (TAG) at this site. The mutant viruses are still capable of replication, but do not synthesise hepatitis $B$ e antigen (HBeAg). They are associated with at least two clinical pictures. In most situations, particularly in the Mediterranean and Far East, an aggressive, protracted form of chronic hepatitis develops. This occurs during the course of an $\mathrm{HBeAg}$ positive infection and probably reflects selection for the $\mathrm{HBeAg}$ negative pre-core mutant. In other patients fulminant hepatitis has been associated with the viral mutant. ${ }^{57}$ In some of these cases it has been shown that the presumed contact of the patient has the mutant strain as a sole or dominant form, suggesting an initial infection with the mutant as a cause of the fulminant hepatitis, rather than selection from a mixed infection. ${ }^{57}$

In view of the medical importance of this viral mutant rapid detection methods for the mutant will be very useful. We have thus developed a procedure for detecting the mutant virus using a variation of the polymerase chain reaction-allele specific PCR. This procedure depends on the discovery that the allele specificity of a PCR primer is determined predominantly by its 3 -end. ${ }^{8}$ Amplification is much reduced if the 3'-terminal is not precisely complementary to its target sequence, even though the rest of the primer sequence is. By designing primers which have a match or mismatch at the 3'-nucleotide, for either the wild type or mutant virus at position 1896 of the HBV genome, amplification can be made highly specific for either virus type. This technique has the advantage that it is based on the PCR and allows investigation to be carried out on formalin fixed, paraffin wax embedded liver biopsy specimens, rather than fresh material.

\section{Methods}

LIVER SAMPLES

Formalin fixed, paraffin wax embedded liver tissues were retrieved from the archives of the Nuffield Department of Pathology and Bacteriology, and the Institute of Pathology, Queen Elizabeth Hospital, Hong Kong. The diagnosis of acute and fulminant hepatitis B was based on histological criteria and serological assay for hepatitis B surface antigen (HBsAg). HBV infection was further confirmed by amplifying a fragment of the precore/core region of the $\mathrm{HBV}$ genome using a
Correspondence to: Dr K A Fleming Accepted for publication 20 January 1992 
Figure 1 Relative locations of primers.

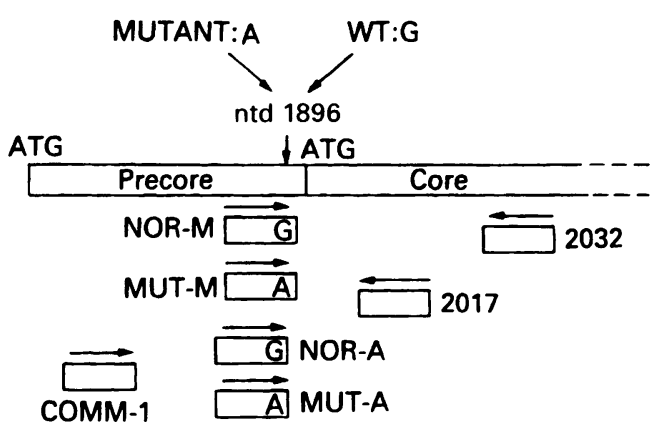

heminested polymerase chain reaction system using primers COMM-1/2032 (40 cycles) followed by COMM-1/2017 (25 cycles).

PRIMERS

All primers were synthesised on an Applied Biosystems 380A DNA synthesiser. Apart from "conventional" amplification primers COMM-1, 2017, and 2032 (relative locations shown in fig 1 , sequences in the table), we also constructed "mutagenesis" and "allele specific" primers which are described below.

\section{Mutagenesis primers}

To construct control templates with either the wild type or mutant sequences with regard to position 1896, two mutagenesis primers were designed (fig 1). Primer NOR-M and MUT-M were synthesised containing sequences corresponding to the wild type ("normal") and mutant sequences, respectively. The suffix $M$ signifies a mutagenesis primer.

To generate the wild type or mutant product, $100 \mathrm{pg}$ of a plasmid containing a full length HBV insert ( $\mathrm{pHBV} 130^{\circ}$ ) was amplified by PCR using either NOR-M/2032 or MUT$M / 2032$. Forty cycles with a thermal profile of $94^{\circ} \mathrm{C}$ for one minute, $55^{\circ} \mathrm{C}$ for one minute, and $72^{\circ} \mathrm{C}$ for one minute were performed.

\section{Allele specific primers}

NOR-A and MUT-A were allele specific primers synthesised corresponding to the wild type and mutant sequences, respectively. The suffix A signifies an allele specific primer. An additional mismatch was introduced at position 2 from the 3'-end of each primer to increase further the specificity of the primers (underlined in the table).

POLYMERASE CHAIN REACTION

PCR was carried out as described, ${ }^{1}$ using reagents from a GeneAmp DNA amplification reagent kit (Perkin-Elmer Cetus) in $100 \mu \mathrm{l}$

Primer sequences

\section{"Conventional" primers.}

COMM-1: 5'CACCTCTGCCTAATCATCTCATGTTCATGT3'

2032: 5'CTGACTACTAATTCCCTGGATGCTGGGTCT

2017: 5'ATGGGATCCCTGGATGCTGGGTCTTCCAAA 3 '

Mutagenesis primers:

NOR-M: 5'CTCCAAGCTGTGCCTTGGGTGGCTTTGGGGCAT3'

MUT-M: 5'CTCCAAGCTGTGCCTTGGGTGGCTTTAGGGCAT3'

Allele specific primers:

NOR-A: 5'CTCCAAGCTGTGCCTTGGGTGGCTGTG3'

MUT-A: 5'CTCCAAGCTGTGCCTTGGGTGGCTGTA3'

Primers 2032 and 2017 were described by Kaneko et al. " Primers COMM-1, NOR-M, MUT-M, NOR-A and MUT-A were designed from published sequences. ${ }^{12}$ The nucleotides in bold in NOR-M and MUT-M denote the base at position 1896. Deliberate mismatches to increase specificity of allele-specific primers NOR-A and MUT-A are underlined. reactions. Thermal cycling was performed on a Biometra TRIO Thermoblock. Individual cycling profiles are described under the relevant sections. Preparation of formalin fixed, paraffin wax embedded liver tissues for PCR was as described, ${ }^{10}$ except that the dewaxed tissues were digested with proteinase $\mathrm{K}$ (200 $\mu \mathrm{g} / \mathrm{ml}$; Boehringer Mannheim) overnight at $37^{\circ} \mathrm{C}$ in a digestion buffer containing $50 \mathrm{mM}$ TRIS, pH 8.5, $1 \mathrm{mM}$ EDTA, $0.5 \%$ Tween 20 (Sigma). Ten microlitres were used per PCR. For heminesting, $2 \mu \mathrm{l}$ of the first round PCR product (using COMM-1/2032) was reamplified in a second round reaction using COMM-1/2017.

GEL QUANTIFICATION OF PCR PRODUCT

For quantification, $2 \mu \mathrm{l}$ of the relevant PCR product-that is, either the 291 base pair reference product (using MUT-M/2032 or NOR-M/2032), or the 329 base pair second round PCR product (using primers COMM1/2017) - was run on an ethidium stained gel. Serial dilutions $(4 \mu \mathrm{g}, 2 \mu \mathrm{g}, 1 \mu \mathrm{g}, 0.5 \mu \mathrm{g}$ and $0.25 \mu \mathrm{g})$ of pBR322 DNA digested with $M s p \mathrm{I}$ were run adjacent to the PCR product to be quantified. An estimation of the amount of the HBV PCR product was made by comparison with the 309 base pair band of the pBR322 MspI marker (corresponding to $280 \mathrm{ng}, 140$ $\mathrm{ng}, 70 \mathrm{ng}, 35 \mathrm{ng}$ and $17.5 \mathrm{ng}$ in the respective dilution).

\section{Results}

GENERATION OF CONTROL TEMPLATES

PCR products with either the wild type or mutant sequence at position 1896 were produced using NOR-M/2032 and MUT$M / 2032$, respectively (fig 2 ). These PCR products were quantified by comparison with known standards on an ethidium stained agarose gel. After appropriate dilution, $10^{8}$ molecules of these PCR products were used in subsequent amplification by allele specific PCR.

ALLELE SPECIFIC PCR

The sequence specificity of the primers for the wild type and mutant viruses (NOR-A and MUT-A, respectively) was tested using a standard assay using $10^{8}$ control template molecules with either the wild type or mutant sequence. As sequence specificity of PCR primers is critically dependent on the annealing temperature, we systematically investigated annealing temperatures ranging from $63^{\circ} \mathrm{C}$ to $71^{\circ} \mathrm{C}$. At each temperature tested, four reactions were carried out: MUT-A/2017 on the mutant template; NOR-A/2017 on the mutant template; MUT-A/2017 on the wild type template; and NOR-A/2017 on the wild type template. At an annealing temperature of $70^{\circ} \mathrm{C}$, the reaction was very specific (fig 3 ). This condition was used for subsequent study.

ALLELE SPECIFIC PCR ON CLINICAL SAMPLES The degree of allele specificity in PCR is determined by the amount of starting tem- 


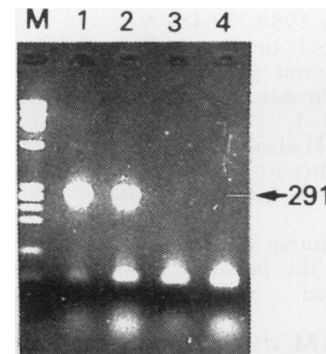
reference PCR templates using mutagenesis primers. Lane $1=M U T-M / 2032$ on $\mathrm{pHBV} 130$, lane $2=N O R-M / 2032$ on pHBV130, lane $3=$ MUT-M $/ 2032$ on water (negative control), lane $4=$ NOR-M/2032 on water (negative control). Arrow marked 291 indicates position of the 291 bp PCR product. $M$ denotes $\varphi$ X174 DNA digested with HaelII (marker).
Figure 2 Generation of

plate. However, in clinical specimens infected by $\mathrm{HBV}$ the number of virus molecules may vary from case to case. This variability may affect the specificity of our allele specific PCR assay. We have thus adopted a strategy whereby we first amplified the HBV DNA from a clinical specimen by heminested PCR. This initial "amplification phase" was then followed by quantification of the PCR product by comparison with known standards after electrophoresis on an ethidium stained agarose gel. Product $\left(10^{8}\right.$ molecules) were then subjected to a "diagnostic phase" using allele specific PCR. This two-step approach is illustrated in fig 4. As the starting material for the "diagnostic phase" is a PCR product, the system is essentially analogous to the one we used for the establishment of allele specificity of the primers.

To provide an internal control during the "diagnostic phase" allele specific PCR, we included a third primer COMM-1 in all allele specific amplifications. COMM-1 and 2017 primed the synthesis of a 329 base pair PCR product which acted as a positive control. MUT-A/2017 and NOR-A/2017 would direct amplification from the mutant and wild type viruses, respectively.

As a preliminary investigation using the allele specific PCR assay, we chose three patients with fulminant hepatitis B (two from Hong Kong and one from Oxford) and four patients with acute hepatitis B from Hong Kong. PCR was carried out from paraffin wax embedded liver biopsy specimens, as outlined. Following the "amplification phase", a 329 base pair HBV-PCR product was visible from all seven cases (results not shown). After the "diagnostic phase" only the wild type viral sequence was detected in all seven patients (fig 5). The 329 base pair internal positive control was visible in all tracks, thus excluding amplification failure as the reason behind our inability to detect mutant $\mathrm{HBV}$ in the three cases of fulminant hepatitis.
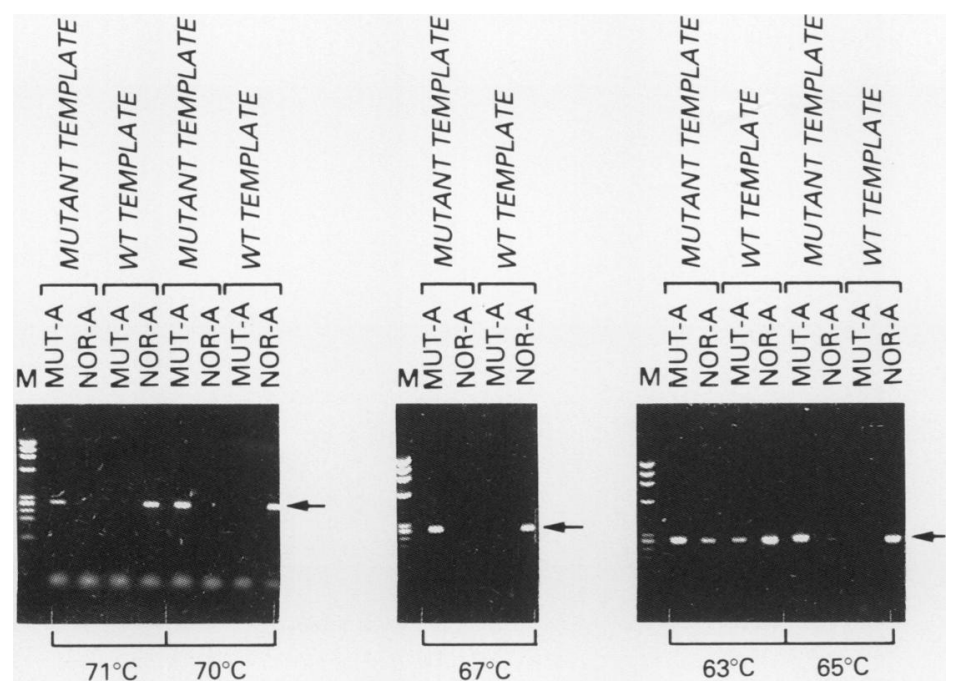

Figure 3 Temperature titration for allele specific PCR assay. $10^{8}$ molecules of a reference mutant or wild type (marked above each lane) template was used for each PCR. The cycling profile was $94^{\circ} \mathrm{C}$ for one minute, annealing at temperature marked below the gel for one minute, and extension at $72^{\circ} \mathrm{C}$ for one minute for 15 cycles. MUT- $A$ and NOR-A indicate reactions carried out using MUT-A/2017 and NOR-A/2017, respectively. Arrow marks position of 285 base pair PCR product. M denotes $\varphi X 174$ DNA digested with HaeIII (marker).

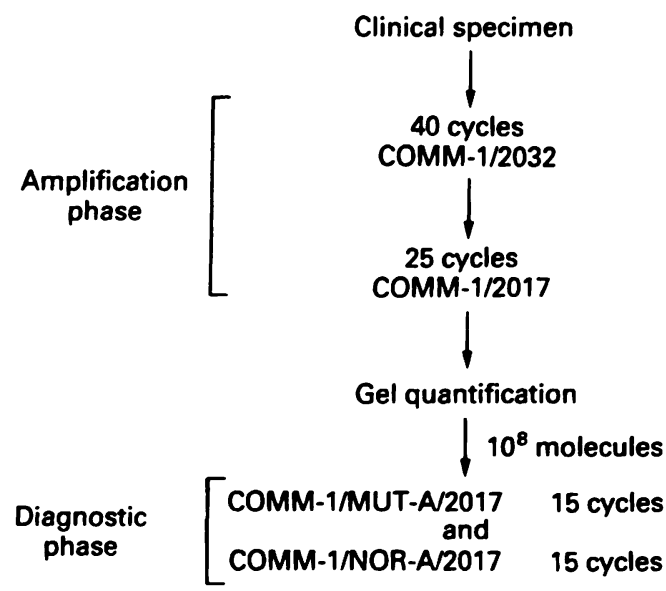

Figure 4 Two-step approach for using allele specific PCR on clinical samples.

\section{Discussion}

Compared with previously described methods for the detection of the pre-core HBV mutant such as sequencing ${ }^{25.7}$ and allele specific oligonucleotide (ASO) hybridisation, ${ }^{13}$ our method is rapid and completely non-radioactive. The ability of the technique to use paraffin wax embedded tissues as starting material suggests that the method may be used for large scale retrospective analysis of the role of the mutant in a variety of liver diseases.

The use of a two-step approach involving an "amplification phase" followed by a "diagnostic phase" overcomes several difficulties of applying an allele specific PCR assay to formalin fixed, paraffin wax embedded tissues. First, the exact amount of amplifiable nucleic acid present in paraffin wax embedded tissues may vary from case to case and these tissues may amplify with varying efficiencies. ${ }^{1014}$ Second, the viral titre may also differ from patient to patient. Both of these problems are solved by the two-step approach in which the "amplification phase" is aimed at amplifying the viral

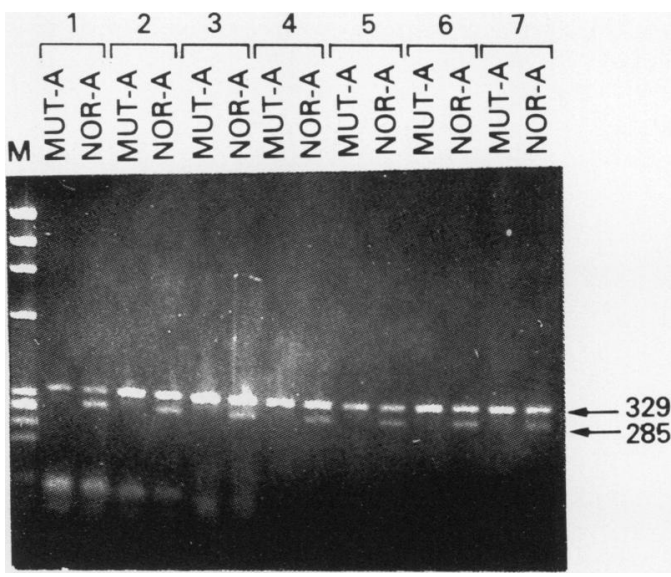

Figure 5 Allele specific PCR on clinical samples. Cases 1-3 were fulminant hepatitis $B$ and cases 4-7 were acute hepatitis B. MUT-A and NOR-A represent reactions carried out using COMM-1/MUT-A/2017 and COMM-1/NOR-A/2017, respectively. 285 and 329 indicate positions of the allele specific PCR product (using MUT-A or NOR-A/2017) and the internal control (using COMM-1/2017), respectively. M denotes $\varphi X 174 D N A$ digested with HaeIII (marker). 
molecules present in a tissue section to a PCR plateau. This is followed by gel quantification and then a pre-determined amount of the PCR product is then subjected to a "diagnostic phase" which has been calibrated to handle this quantity of product. This two-step strategy should be suitable to any use of allele specific PCR to paraffin wax embedded tissues and thus may have important uses in other diagnostic situations.

In our preliminary series of three cases of fulminant hepatitis $B$, only the wild type virus was found. While this contradicts the published findings of the role of the mutant virus in a number of Japanese ${ }^{56}$ and Israeli $^{7}$ cases of fulminant hepatitis $B$, our results are similar to the data reported in cases from the USA. ${ }^{15}$ Taken together, these data suggest a geographical variation in the involvement of precore HBV mutants in fulminant hepatitis B. We are currently undertaking a larger study to establish the generality of these observations. The rapidity of the allele specific PCR assay should make this goal attainable.

With the accumulation of sequence information on HBV and other viruses, increasing numbers of viral variants will be discovered. Allele specific PCR is an efficient method for the detection of these viral variants.

We thank the Wellcome Trust and Foulkes' Foundation Fellowship for financial support.

1 Saiki RK, Gelfand D, Stoffel S, et al. Primer-directed enzymatic amplification of DNA using a thermostable
DNA polymerase. Science 1988;239:487-91.

2 Brunetto MR, Stemler M, Schodel F, et al. Identification of HBV variants which cannot produce precore derived $\mathrm{HBeAg}$ and may be responsible for severe hepatitis. ItaI $f$ Gastroenterol 1989;21:151-4.

3 Carman WF, Jacyna MR, Hadziyannis $S$, et al. Mutation preventing formation of "hepatitis B e antigen" in patients preventing formation of "hepatitis B e antigen" in patients with 980 .

4 Galibert F, Mandart E, Fitoussi F, Tiollais P, Charney P Nucleotide sequence of the hepatitis $B$ virus genome subtype ayw) cloned in $E$. coli. Nature 1979;281:646-50

5 KosakaY, Takase K, Kojima M, et al. Fulminant hepatitis B Induction by hepatitis $B$ virus mutants defective in the precore region and incapable of encoding e antigen. Gastroenterology 1991;100:1087-94.

6 Omata M, Ehata T, Yokosuka O, Hosoda K, Ohto M. Mutations in the precore region of hepatitis $B$ virus DNA in patients with fulminant and severe hepatitis. $N$ Engl $f$ Med 1991;324:1699-704.

7 Liang TJ, Hasegawa K, Rimon N, Wands JR, Ben-Porath E A hepatitis $B$ virus mutant associated with an epidemic of A hepatitis B virus mutant associated with an epidem

8 Newton CR, Graham A, Heptinstall LE, et al. Analysis of any point mutation in DNA. The amplification refractory mutation system (ARMS). Nucleic Acids Res 1989;17:2503-16.

9 Gough NM, Murray K. Expression of the hepatitis B virus surface, core and e antigen genes by stable rat and mouse cell lines. 7 Mol Biol 1982;162:43-6.

10 LoY-MD, Mehal WZ, Fleming KA. In vitro amplification of hepatitis B virus sequences from liver tumour DNA and from paraffin wax embedded tissues using the polymerase from paraffin wax embedded tissues using the

11 Kaneko S, Feinstone SM, Miller RH. Rapid and sensitive method for the detection of serum hepatitis B virus DNA method for the detection of serum hepatitis B virus DNA
using the polymerase chain reaction technique. $f$ Clin Microbiol 1989;27:1930-3.

12 Ono Y, Onda H, Sasada R, Igarashi K, Sugino Y, Nishioka $K$. The complete nucleotide sequences of the cloned hepatitis B virus DNA; subtype adr and adw. Nucleic Acids Res 1983;11:1747-57

13 Brunetto MR, Giarin MM, Oliveri F, et al. Wild-type and e antigen-minus hepatitis $B$ viruses and course of chronic hepatitis. Proc Natl Acad Sci USA 1991;88:4186-90.

14 An SF, Fleming KA. Removal of inhibitor(s) of the polymerase chain reaction from formalin fixed, paraffin polymerase chain reaction from formalin fixed, paraf

15 Hasegawa K, Shapiro CN, Alter MJ, Liang TJ. Lack of an association of hepatitis B virus precore mutations with fulminant hepatitis B in USA. Hepatology 1991;14:78A. 